\title{
T20K: An Immunomodulatory Cyclotide on Its Way to the Clinic
}

\author{
Carsten Gründemann ${ }^{1}\left[\right.$ ] $\cdot$ Kjell G. Stenberg ${ }^{2} \cdot$ Christian W. Gruber $^{3}[$
}

Accepted: 24 March 2018 / Published online: 5 April 2018

(c) The Author(s) 2018

\begin{abstract}
Cyclotides represent a new class of natural plant compounds, which could be considered ideal "template" molecules for drug design. Their structure is characterized by a circular cystine-knot motif, comprising an amino acid chain stabilized by three conserved disulfide bonds in a knotted arrangement, and a head-to-tail cyclized peptide backbone. Our laboratories initially demonstrated their activity to modulate immune cell signaling. Following nearly a decade of extensive research, we are now aware that cyclotide-based peptides provide an outstanding opportunity to develop novel drugs for autoimmune disorders, especially multiple sclerosis. Here we review and demonstrate the potential of cyclotide-derived peptide therapeutics on their way from preclinical studies to clinical trials as promising therapeutics in immunopharmacological applications.
\end{abstract}

Keywords Cyclotide $\cdot$ Multiple sclerosis $\cdot$ Immunosuppression $\cdot$ Circular peptide

\section{Introduction}

Multiple sclerosis (MS) is the most common autoimmune disease affecting the central nervous system (CNS) (McFarland and Martin 2007; Ransohoff et al. 2015). It is characterised by auto-reactive T-cells that induce demyelination and neuronal degradation. Treatment options are limited, and several MS medications need to be administered by parenteral application and only show modest clinical efficacy. Oral active drugs such as fingolimod have been associated with safety concerns (Ransohoff et al. 2015). Consequently, there is a high unmet medical need for novel, less toxic and especially orally (patient-friendly) active therapeutics. Nature offers an abundance of compounds for drug discovery. Recently, we have shown that the circular plant peptide kalata B1 (kB1) is able to silence T-cell proliferation

Carsten Gründemann

carsten.gruendemann@uniklinik-freiburg.de

Christian W. Gruber

christian.w.gruber@meduniwien.ac.at

1 Institute for Infection Prevention and Hospital Epidemiology, Center for Complementary Medicine, Faculty of Medicine, University of Freiburg, Freiburg, Germany, Breisacher Str. 115B, 79111 Freiburg, Germany

2 Cyxone AB, Adelgatan 21, 21122 Malmö, Sweden

3 Center for Physiology and Pharmacology, Medical University of Vienna, Schwarzspanierstrasse 17, 1090 Vienna, Austria in vitro via an interleukin-2 (IL-2) dependent mechanism (Gründemann et al. 2013). Encouraged by these observations, we developed our lead compound cyclotide [T20K] kB1 ('T20K') (Fig. 1) and tested it in the MS mouse model of experimental autoimmune encephalomyelitis (EAE). We were able to show that treatment of mice with the cyclotide resulted in a significant delay in the onset of clinical observations and diminished symptoms of EAE upon parenteral or oral prophylactic administration. Cyclotide application substantially impeded disease progression and exhibited moderate adverse effects (Thell et al. 2016). Considering their stable structural topology and oral activity, cyclotidebased peptides appear to be good candidates for pharmaceutical drug development for treatment of diseases with an overreactive immune system.

In this review, we would like to (i) provide a brief background on cyclotides, (ii) summarize past efforts investigating their immunosuppressive properties in cell culture and in animal models, (iii) discuss insights about ongoing preclinical and early clinical development studies with T20K and (iv) evaluate the future potential of this peptide drug candidate as novel therapeutic intervention for autoimmune disorders.

\section{Cyclotides and Their Immunomodulatory Properties}

Natural products play a pivotal role in modern drug discovery (Koehn and Carter 2005), and they continue to provide 


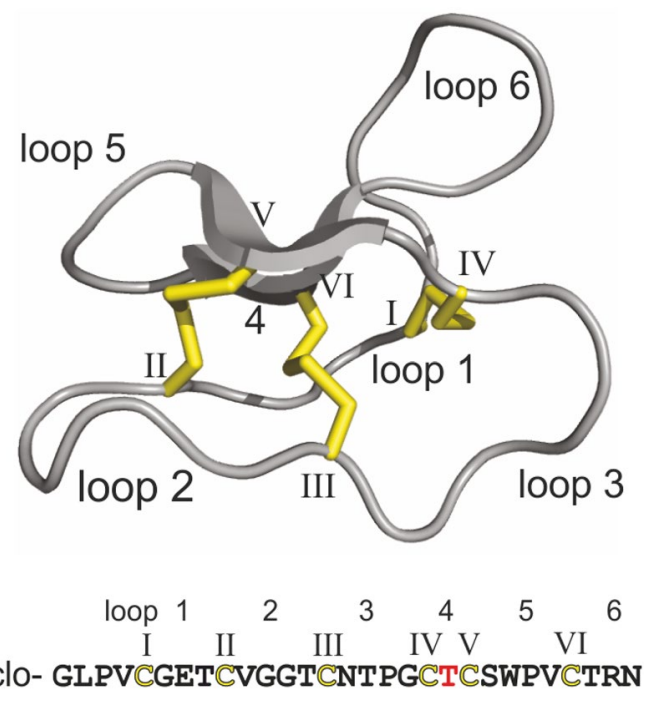

Fig. 1 Amino acid sequence and structural model of the cyclotide T20K. The structure of [T20K]kalata B1 (T20K) is shown in cartoon form. The six conserved cysteines are labeled with roman numerals and the cystine-knot disulfide connectivity $\left(\mathrm{C}_{\mathrm{I}}-\mathrm{C}_{\mathrm{IV}}, \mathrm{C}_{\mathrm{II}}-\mathrm{C}_{\mathrm{V}}\right.$ and $\left.\mathrm{C}_{\mathrm{III}}-\mathrm{C}_{\mathrm{VI}}\right)$ is indicated. The amino acid sequence and the loops of the peptide backbone are shown. The position of the threonine to lysine replacement in the synthetic kalata B1 analogue is shown (as red letter). This representation was prepared using PyMol based on the three-dimensional solution structure of kalata B1 (pdb code: 1NB1). Reproduced with permission from the Creative Commons Attribution License (Gründemann et al. 2013). (Color figure online)

innovative lead compounds currently entering clinical trials (Harvey et al. 2015). The increasing interest for peptidebased drugs has boosted development of nature-derived peptides for therapeutic applications (Craik et al. 2013; Kaspar and Reichert 2013). The major drawbacks of most peptide pharmaceuticals are their low stability and lack of oral activity which limits their clinical use (Craik et al. 2013).

Recently we demonstrated immunosuppressive effects of the circular peptide kB1 (Gründemann et al. 2012, 2013). This plant-derived peptide belongs to the family of cyclotides, well known for their cyclic cystine-knot topology (Craik 2001). This unique three-dimensional fold confers them intrinsic stability to resist chemical, enzymatic and thermal degradation (Colgrave and Craik 2004). Therefore cyclotides have become attractive tools in chemical biology and drug development (Huang et al. 2015), for instance as template for molecular grafting applications (Craik et al. 2012) and for receptor ligand design (Koehbach et al. 2013), since they exhibit activity following oral administration (Wong et al. 2012).

We demonstrated that cyclotides, in particular T20K, inhibit T-cell proliferation by downregulation of IL-2 release as well as IL-2R/CD25 surface expression and effector activity performance (Gründemann et al. 2013; Thell et al. 2014). The cytokine IL-2 physiologically plays an important role in
T-lymphocyte activation and acts as autocrine factor to stimulate T-cell proliferation (Malek and Bayer 2004). Enhanced or continuous T-cell activation is a major cause of autoimmune disorders, and can lead to persistent inflammation causing tissue and organ damage (Langston et al. 2015). MS is the most common type of autoimmune disease in young adults, which is characterised by sustained inflammation of the CNS. Autoreactive T-lymphocytes $\left(\mathrm{T}_{\mathrm{H}} 17\right)$ target myelin brain antigens eliciting inflammatory cell influx into the CNS, demyelination, axonal damage and neuronal degradation (Hickey 1999; McFarland and Martin 2007). Several therapeutics targeting different aspects to modulate or suppress T-cell signalling are available, but the requirement for parenteral administration route of many drug candidates thus reduces their attractiveness for chronic treatment (Van der Walt et al. 2010). Only three marketed compounds that are specific for MS treatment are active via oral administration, i.e. dimethyl fumarate (Tecfidera ${ }^{\circledR}$ ), teriflunomide (Aubagio $\left.{ }^{\circledR}\right)$ and fingolimod (Gilenya $\left.{ }^{\circledR}\right)$; however, many severe side effects limit their long-term clinical use (Ransohoff et al. 2015).

The remarkable stability and hydrophobic surface properties of cyclotides render them well suited for oral administration (Wong et al. 2012). Our data demonstrate significant therapeutic effects of T20K in the in vivo gold-standard model for MS, the murine EAE approach, after oral administration (Thell et al. 2016). We investigated their efficacy to reduce the polarisation of pathogenic $T_{H} 17$ cells and the rate of relapse by prophylactic administration of cyclotides before disease induction. Moreover, we analysed the therapeutic application of cyclotides during disease progression, which potently ameliorated the EAE symptoms. Preliminary structure activity relationships (SAR) measurements combined with structural studies led to the conclusion that the observed effects are stereospecific due to a direct cyclotidetarget interaction (Gründemann et al. 2012, 2013). Using a chemical proteomics strategy including synthesis and validation of chemical probes, the family of 14-3-3 proteins were identified as a possible direct target of T20K (Hellinger et al. 2017). 14-3-3 proteins are ubiquitous adaptors that play a pivotal function in cell physiology. Hence, strategies to knock out or silence these proteins are difficult and the detailed in vivo mechanism of the cyclotide-14-3-3 interaction remains unknown.

Using a different approach, it was possible to design and synthesize novel grafted cyclotide analogues with activity in the EAE mouse model. The goal to implement molecular grafting was to design molecules that retain both, the activity of an MS-related antigen [myelin oligodendrocyte glycoprotein (MOG)] and the structure and stability of the scaffold onto which the epitope is grafted (Wang et al. 2014).

Beside the above mentioned strategies, cyclotides have thus far been discovered in different plant families. By using 
an ethnobotanical and bioassay-fractionation-based combinatorial approach, the first immunosuppressive cyclotide we discovered was $\mathrm{kB} 1$ isolated from the coffee-family plant Oldenlandia affininis (Gründemann et al. 2012). Based on these data, T20K was defined by using chemical modification strategies as a highly active immunosuppressive cyclotide (Gründemann et al. 2013) demonstrating that such strategy represents an innovative approach to design new leads with immunomodulatory capacities. Along this line, Viola tricolor is a well-known and documented medicinal plant and its traditional use as an herbal remedy against inflammatory immune diseases is widespread all over Europe. Interested to understand the molecular basis for its use in phytomedicine, we characterized the influence of an aqueous Viola extract and defined the presence of cyclotides as active immunosuppressive ingredients (Hellinger et al. 2014). These results demonstrate that combining traditional and modern innovative strategies will open new avenues for the application of cyclotides in immune-related disorders.

\section{Autoimmune Disease and Multiple Sclerosis}

Autoimmune diseases represent an increasing problem in the Western World. With the global incidence and prevalence of autoimmune diseases rising, there is an escalating demand for new products. The autoimmune diseases therapeutics market in the leading geographies (Australia, Japan, USA, UK and Western Europe) show that the market, comprising all the diseases above, was worth $\$ 16.9$ billion in 2002 and grew to $\$ 30.2$ billion in 2010 . It was forecasted to reach $\$ 61.4$ billion in 2017.

MS is the most common autoimmune disease affecting the CNS. It imposes substantial socio-economic burdens on patients, their families and society. There are few therapeutic modalities available, yet unattractive parenteral application or severe side effects remain serious issues. We discovered that the cyclotide $\mathrm{T} 20 \mathrm{~K}$ inhibited the growth of primary cells of the human immune system, and has therapeutic effects in the murine EAE disease model of human MS (Thell et al. 2016). Ongoing experiments hereby suggest that T20K could be a potential candidate for relapsing-remitting MS (RRMS) due to T20K's significant therapeutic effects upon prophylactic administrations to the animals. The RRMS market is dominated by drugs becoming generic, which are associated with considerable side effects limiting their longterm use. Some of the more recent drugs offer symptom relief in patients' refractory to older drugs but are typically associated with pronounced side effects. The reversible T-cell inhibition mechanism of cyclotides (Gründemann et al. 2013) makes them very appealing from an efficacy and safety perspective and it is likely that such products will hold a very competitive position on the MS market.
Furthermore, other autoimmune diseases such as rheumatoid arthritis or organ-specific autoimmune diseases could potentially benefit from novel cyclotide-based therapeutics with immunosuppressive properties.

\section{Status-Quo, Ongoing Preclinical Studies}

For a successful clinical application of T20K, it is essential to define the magnitudes of biological efficacy and toxicity profile to estimate the safety margin of this therapeutic compound. Several preclinical studies from different disciplines have to be combined to answer this question. Hereby, the "dreamland" scenario is that biological and/or predicted clinical efficacy is observed at doses far below toxicity. However, practice usually shows that the biological activity of drug candidate is often at doses below, but close to toxicity (and far away from lethality). The optimal scenario to define a safe zone for clinical use is to identify marker molecules in non-invasive patient samples, which indicate a potential biological/toxic effect, but remain without any adverse clinical signs of toxicity. From previous and ongoing kinetic experiments with $\mathrm{T} 20 \mathrm{~K}$, we have shown a pronounced upregulation of molecules, which have the potential as safety markers. Initially, we started experiments with intravenous (i.v.) and intraperitoneal (i.p.) applications of $\mathrm{T} 20 \mathrm{~K}$ to establish the relationship between administered doses, amounts of T20K in blood and tissues and their effects in animals to help guiding progression to human clinical trials (phase I clinical evaluation). Once this connection has been established, this information can be used to switch over to an oral application (p.o.) and further the development of an oral formulation of T20K for forthcoming clinical phase II studies.

Single administration of T20K to healthy animals did not exhibit toxic effects up to the doses of $15 \mathrm{mg} / \mathrm{kg}$ (i.v.), $75 \mathrm{mg} / \mathrm{kg}$ (i.p.) and $250 \mathrm{mg} / \mathrm{kg}$ (p.o.). This provides an excellent safety margin considering the effective dose level i.p. or p.o. of $1-10 \mathrm{mg} / \mathrm{kg}$ in the mouse model of MS. Ongoing dose range finding studies and formal clinical trial application (CTA)-/investigational new drug (IND)-enabling toxicology studies using the i.v. application route in mice and in dogs will define the nature of T20K-induced toxicity/ tolerability, the maximum tolerated dose (MTD) and overall safety assessment of T20K leading to selection of a safe starting dose for first-in-human clinical trials.

Initial studies strongly suggest a link between $\mathrm{T} 20 \mathrm{~K} \mathrm{C}_{\max }$ (rather than exposure) and acute severe toxicity (animal death). Upon systemic administration, T20K shows a classical biphasic pharmacokinetic profile, specified by initial rapid clearance from the blood and long terminal half-life at biologically irrelevant blood concentrations. No significant differences between plasma and whole blood pharmacokinetics were detected and a pronounced and long-lasting 
tissue distribution is observed in selected organs with main targets in kidney, spleen, liver and intestine. Interestingly, no detectable distribution in the brain or muscle was identified. An upcoming biodistribution study with oral administration of T20K should clarify the observations of a clinical improvement of MS symptoms in the murine EAE model in the absence of detectable free concentrations of T20K in plasma. On a parallel track, we assess mutagenicity and/ or clastogenicity potential as well as neurological effects of $\mathrm{T} 20 \mathrm{~K}$. An in vitro assessment of potential safety liabilities of T20K was done using a high-throughput screening of several drug target panels, including ion channels, transporters, G protein-coupled receptors (GPCRs), nuclear receptors, kinases, and non-kinase enzymes. We determined binding (affinity) and activity (efficacy, potency) on these target panels. It has been established that $\mathrm{T} 20 \mathrm{~K}$ binds to and modulates signaling of certain GPCRs, which is in line with previous observations (Fahradpour et al. 2017; Koehbach et al. 2013), with no association to potential toxicity. It will certainly be interesting to follow up on these studies to investigate possible target proteins of $\mathrm{T} 20 \mathrm{~K}$.

\section{Outlook}

Despite intensive research, the detailed molecular mechanisms of immunosuppressive cyclotides and T20K have not been fully elucidated, yet. Applying chemical pharmacology strategies to reveal the mechanism of action or a target protein generated some promising results, but the interaction of $14-3-3$ and T20K needs to be further studied. Also it is important to analyse any interactions of $\mathrm{T} 20 \mathrm{~K}$ with cellular transmembrane proteins by using target-affinity pull downs, and functional signalling assays to analyse 'hits' from the pharmacodynamics screen in more detail. From previous work, we designed and engineered immunosuppressive inactive cyclotide variants which differ from T20K by the replacement of only one amino acid. Comparing side-byside with T20K, these represent optimal tools to identify a defined target by using 'omics' technologies.

For optimal development of clinical trials, it will also be important to identify and characterise relevant biomarkers. These are markers-ideally being detected in blood samples-which could be considered as non-invasive early indicators of molecular activity, potential clinical efficacy and/ or upcoming toxicity. For instance, from preclinical studies, we have identified a set of interesting candidate cytokines which expression becomes affected upon $\mathrm{T} 20 \mathrm{~K}$ administration, and we are currently investigating the modulation of their expression profile in more details.

To consider further development of an orally effective drug in the clinics, it is imperative to establish a full biodistribution profile of T20K upon p.o. administration, in comparison with i.v. route of administration. Although this will not be required for currently planned phase I development with an i.v. formulation, eventually the company intends to develop an oral drug, and hence we are already committed to drive this development further, also working on suitable and enhanced oral formulations that will be needed for early stages of clinical development.

Finally, in the recent past, we have established together with a contract manufacturing organization (CMO) a rational method for large-scale synthesis of T20K. With specialized contract research organizations (CROs), we developed a sensitive bioanalytical method to detect T20K in plasma and in various tissues and finally optimized drug stability by development of several formulations of T20K. Currently, we are developing an optimized oral formulation to perform first pilot studies as well as a feasibility study is ongoing to increase the production process of T20K to have an economic strategy to fulfil its need for a successful and progressive path into the clinic.

Acknowledgements Open access funding provided by Austrian Science Fund (FWF). We would like to thank Dr. Michel Janicot (JMi ONConsulting) for comments on the manuscript. Work in the laboratory of C.G. has been financially supported by the Software AG foundation and DAMUS-DONATA e.V. C.W.G has been financially supported by the Austrian Science Fund (FWF, I3243 and P24743), the Austria Wirtschaftsservice (AWS-PRIZE 2016, P1308423) and the Australian Research Council (ARC Future Fellowship Scheme, FT140100730).

\section{Compliance with Ethical Standards}

Conflict of interest C.G. and C.W.G. are inventors of the patent "Pharmaceutical composition for treating or preventing disorder e.g. autoimmune disorder, hypersensitivity disorder comprises a cyclotide for use in immunosuppression, optionally carrier, excipient or diluent" (Patent No.: WO2013093045-A2; WO2013093045-A3; AU2012356772A1; CA2859708-A1; EP2793923-A2; US2014369930-A1; US2016039882-A9; RU2014129918-A; US9453052-B2; US2016367626-A1; AU2012356772-B2; US2017239327-A9; AU2017232191-A1), and serve as members of the scientific advisory board of Cyxone AB (http://www.cyxone.com). K.J.S is CEO and shareholder of Cyxone AB.

Ethical Approval All applicable international, national, and/or institutional guidelines for the care and use of animals were followed.

Open Access This article is distributed under the terms of the Creative Commons Attribution 4.0 International License (http://creativeco mmons.org/licenses/by/4.0/), which permits unrestricted use, distribution, and reproduction in any medium, provided you give appropriate credit to the original author(s) and the source, provide a link to the Creative Commons license, and indicate if changes were made.

\section{References}

Colgrave ML, Craik DJ (2004) Thermal, chemical, and enzymatic stability of the cyclotide kalata B1: the importance of the cyclic. 
Cystine Knot Biochem 43:5965-5975. https://doi.org/10.1021/ bi049711q

Craik DJ (2001) Plant cyclotides: circular, knotted peptide toxins. Toxicon 39:1809-1813

Craik DJ, Swedberg JE, Mylne JS, Cemazar M (2012) Cyclotides as a basis for drug design. Expert Opin Drug Discov 7:179-194. https ://doi.org/10.1517/17460441.2012.661554

Craik DJ, Fairlie DP, Liras S, Price D (2013) The future of peptidebased drugs. Chem Biol Drug Des 81:136-147. https://doi. org/10.1111/cbdd.12055

Fahradpour M, Keov P, Tognola C, Perez-Santamarina E, McCormick PJ, Ghassempour A, Gruber CW (2017) Cyclotides isolated from an ipecac root extract antagonize the corticotropin releasing factor type 1 receptor. Front Pharmacol 8:616. https://doi.org/10.3389/ fphar.2017.00616

Gründemann C, Koehbach J, Huber R, Gruber CW (2012) Do plant cyclotides have potential as immunosuppressant peptides? J Nat Prod 75:167-174. https://doi.org/10.1021/np200722w

Gründemann C et al (2013) Cyclotides suppress human T-lymphocyte proliferation by an interleukin 2-dependent Mechanism. PLoS ONE 8:e68016. https://doi.org/10.1371/journal.pone.0068016

Harvey AL, Edrada-Ebel R, Quinn RJ (2015) The re-emergence of natural products for drug discovery in the genomics era. Nat Rev Drug Discov 14:111-129. https://doi.org/10.1038/nrd4510

Hellinger R, Koehbach J, Fedchuk H, Sauer B, Huber R, Gruber CW, Grundemann C (2014) Immunosuppressive activity of an aqueous Viola tricolor herbal extract. J Ethnopharmacol 151:299-306. https://doi.org/10.1016/j.jep.2013.10.044

Hellinger R et al (2017) Chemical proteomics for target discovery of head-to-tail cyclized mini-proteins. Front Chem 5:73. https://doi. org/10.3389/fchem.2017.00073

Hickey WF (1999) The pathology of multiple sclerosis: a historical perspective. J Neuroimmunol 98:37-44

Huang YH, Henriques ST, Wang CK, Thorstholm L, Daly NL, Kaas Q, Craik DJ (2015) Design of substrate-based BCR-ABL kinase inhibitors using the cyclotide scaffold. Sci Rep 5:12974. https:// doi.org/10.1038/srep12974

Kaspar AA, Reichert JM (2013) Future directions for peptide therapeutics development. Drug Discov Today 18:807-817. https://doi. org/10.1016/j.drudis.2013.05.011
Koehbach J et al (2013) Oxytocic plant cyclotides as templates for peptide $\mathrm{G}$ protein-coupled receptor ligand design. Proc Natl Acad Sci USA 110:21183-21188. https://doi.org/10.1073/pnas.1311183110

Koehn FE, Carter GT (2005) The evolving role of natural products in drug discovery. Nat Rev Drug Discov 4:206-220. https://doi. org/10.1038/nrd1657

Langston PK, Yang M, Bierbach U, Parsonage D, Poole LB, Price MJ, Grayson JM (2015) Au-ACRAMTU-PEt3 alters redox balance to inhibit T cell proliferation and function. J Immunol 195:19841994. https://doi.org/10.4049/jimmunol.1400391

Malek TR, Bayer AL (2004) Tolerance, not immunity, crucially depends on IL-2. Nat Rev Immunol 4:665-674. https://doi. org/10.1038/nri1435

McFarland HF, Martin R (2007) Multiple sclerosis: a complicated picture of autoimmunity. Nat Immunol 8:913-919. https://doi. org/10.1038/ni 1507

Ransohoff RM, Hafler DA, Lucchinetti CF (2015) Multiple sclerosis-a quiet revolution. Nat Rev Neurol 11:246. https://doi.org/10.1038/ nrneurol.2015.49

Thell K, Hellinger R, Schabbauer G, Gruber CW (2014) Immunosuppressive peptides and their therapeutic applications. Drug Discov Today 19:645-653. https://doi.org/10.1016/j.drudis.2013.12.002

Thell K et al (2016) Oral activity of a nature-derived cyclic peptide for the treatment of multiple sclerosis. Proc Natl Acad Sci USA 113:3960-3965. https://doi.org/10.1073/pnas.1519960113

Van der Walt A et al (2010) Neuroprotection in multiple sclerosis: a therapeutic challenge for the next decade. Pharmacol Ther 126:82-93. https://doi.org/10.1016/j.pharmthera.2010.01.006

Wang CK et al (2014) Molecular grafting onto a stable framework yields novel cyclic peptides for the treatment of multiple sclerosis. ACS Chem Biol 9:156-163. https://doi.org/10.1021/cb400548s

Wong CT, Rowlands DK, Wong CH, Lo TW, Nguyen GK, Li HY, Tam JP (2012) Orally active peptidic bradykinin B1 receptor antagonists engineered from a cyclotide scaffold for inflammatory pain treatment. Angew Chem 51:5620-5624. https://doi.org/10.1002/ anie. 201200984 